\title{
Acute Coronary Disease in very Young Patient: A Reality!
}

\author{
Erivelton Nascimento, ${ }^{1,2}$ Ana Luisa Freitas e Pena Mello, ${ }^{2}{ }^{\circledR}$ Ludmila Souza da Cunha, $^{2}{ }^{\oplus}$ Marcelo Ferreira Villela $^{3}$ \\ Universidade Federal Fluminense, ${ }^{1}$ Niterói, $R J$-Brazil \\ Centro Universitário de Volta Redonda, ${ }^{2}$ Volta Redonda, RJ - Brazil \\ Hospital São João Batista, ${ }^{3}$ Volta Redonda, RJ - Brazil
}

\section{Introduction}

Acute cardiovascular events such as acute myocardial infarction (AMI) are uncommon in young individuals. ${ }^{1}$ Additionally, it is known that different factors may be involved in the occurrence of AMI. ${ }^{1}$ Here, we report a case of acute myocardial infarction due to severe coronary lesion in a very young patient.

\section{The Case}

P.A.G.T., 22-year-old male, was brought to the hospital with a $\Delta \mathrm{t}$ of 60 minutes experiencing typical chest pain that started during a soccer match and did not radiate. On admission, the patient had high blood pressure $(160 \times 80 \mathrm{mmHg})$ and was diaphoretic. Electrocardiogram (ECG) was done within eight minutes of his arrival and showed elevation of segment ST in leads DII, DIII, AVF and V3 to V5. (Figure 1). Due to clinical findings and ECG tracing, thrombolytic therapy with alteplase was started. He also received double antiplatelet therapy, statin, angiotensin converting enzyme inhibitor and enoxaparin. The patient showed pain relief and serial electrocardiograms showed a reduction of the ST segment elevation greater than $50 \%$, which is criterion for reperfusion. At the end of thrombolysis, the patient experienced sinus bradycardia and blood pressure of $114 \times 61 \mathrm{mmHg}$. Laboratory exams at admission revealed negative qualitative troponin $\mathrm{I}(<0,5$ ng/mL), creatine kinase (CK) of 448/L and CKMB of 28

\section{Keywords}

Young; Artery Coronary Diseases; Hyperlipidemias; Atherosclerosis; Myocardial Infarction; Hereditity; Street Drugs; Statins; Enoxaparin; Platelets/treatment.
U/L. Fourteen hours after admission, laboratory results showed positive qualitative troponin, total CK of 1171 U/L and CKMB of $121 \mathrm{U} / \mathrm{L}$. After 24 hours of the initial event, the patient was transferred to a catheterization lab for invasive test. The coronary angiography revealed descending anterior coronary artery with severe segmental lesion (90\%) with an intracoronary thrombus in its proximal third (Figure 2). Angioplasty was performed, with successful implantation of two conventional stents and final TIMI flow III.

When asked about his family history of cardiovascular diseases, the patient denied family history of premature cardiovascular disease, or use of cocaine, tobacco, or other drugs. Alcohol consumption was of 18 doses per week. The screening for diabetes revealed fasting glucose of $81 \mathrm{mg} / \mathrm{dL}$ and $\mathrm{HbA} 1 \mathrm{c}$ of $5 \%$. The patient also denied any condition that required long term treatment such as immunodeficiency or inflammatory disorders. After five days, the patient was discharged with stable vital signs and no symptoms. Subsequent exams showed total cholesterol $101 \mathrm{mg} / \mathrm{dL}$, low-density lipoprotein 44mg/dL (LDL), high-density lipoprotein (HDL) 37mg/ $\mathrm{dL}$, very low-density lipoprotein (VLDL) 20mg/dL, non-high-density lipoprotein $64 \mathrm{mg} / \mathrm{dL}$ and triglycerides $121 \mathrm{mg} / \mathrm{dL}$. Normal coagulation tests. Biomarkers for coagulopathies were requested. Echocardiogram did not show segmental alterations after hospital discharge.

\section{Discussion}

The risk factors associated with ST-segment elevation myocardial infarction (STEMI) in young adults can be different as compared with elderly population. ${ }^{1,2}$ A study conducted in Switzerland with 27 patients younger than 30 years old with previous STEMI revealed that history of smoking, dyslipidemia and

Mailing Address: Erivelton Nascimento

Rua Marques do Paraná, 303. Postal Code: 24033-900, Niterói, RJ - Brazil

E-mail: hpcrates7@gmail.com 


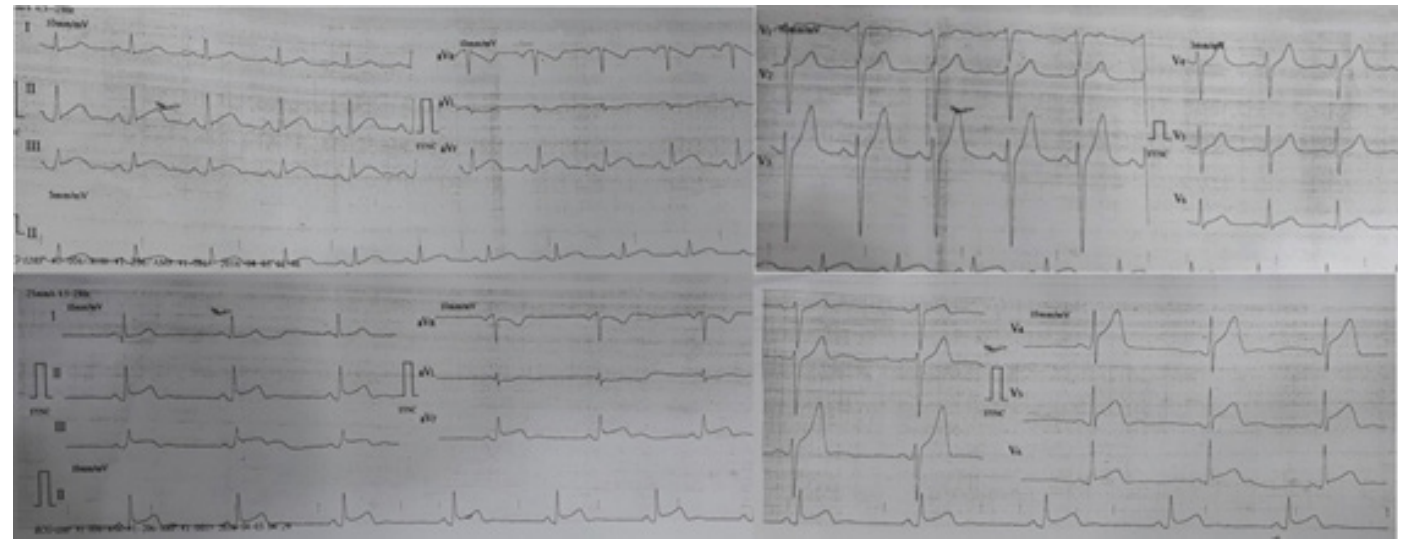

Figure 1 - Electrocardiogram showing inferior and anteroseptal wall ST segment elevation

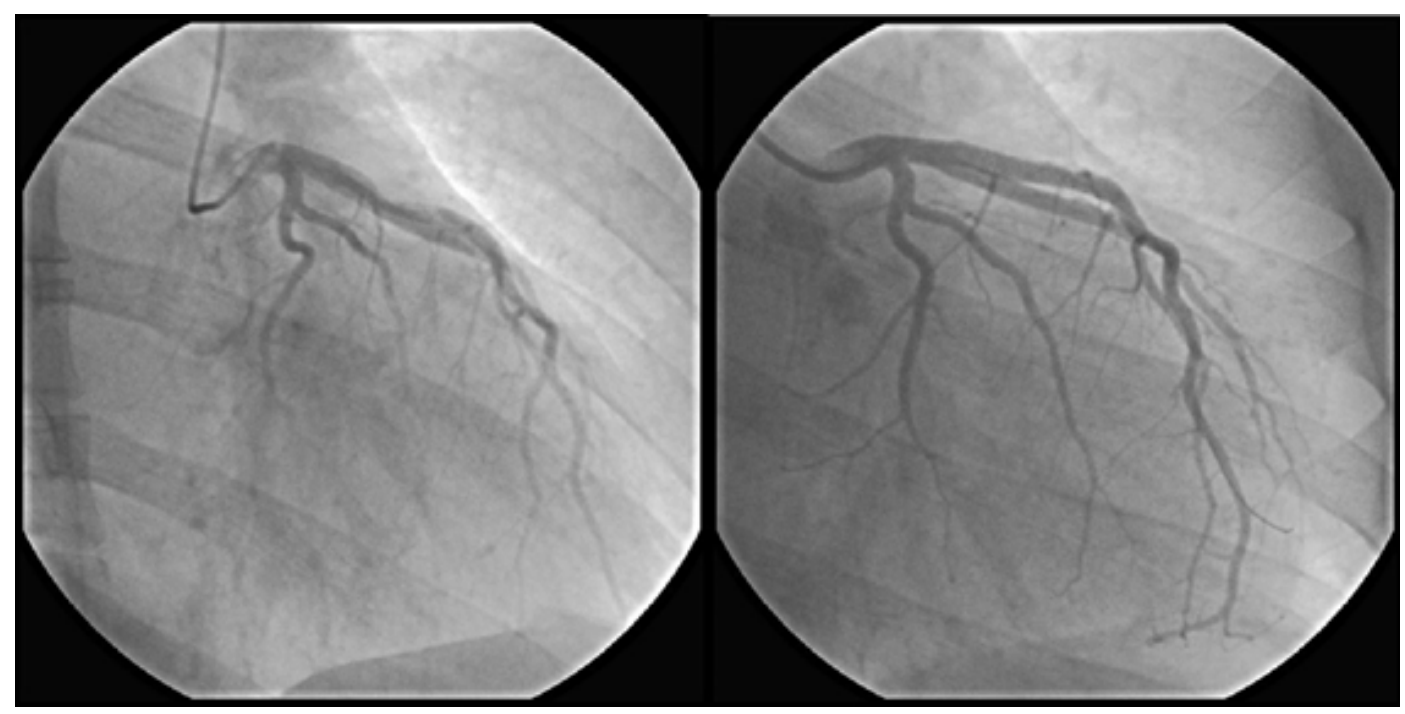

Figure 2 - Coronary angiography images showing severe lesion of anterior descending artery before angioplasty (on the left) and after angioplasty (on the right)

family history of premature cardiovascular diseases ( $<55$ years old for men and $<65$ years old for women) were the most common factors in this group. ${ }^{1}$

A cohort study called AFIJI (Appraisal of risk Factors in young Ischemic patients Justifying aggressive Intervention) investigated 880 patients under 45 years old (average age of 40 years) that experienced an acute coronary syndrome associated with obstructive causes. ${ }^{3}$ Most of the patients were male (87\%). Different from the findings in this report, the prevalent risk factors identified in the AFIJI study were smoking, family history of coronary artery disease (CAD) and hyperlipidemia. ${ }^{3}$

As for the etiology, there are many causes of acute STEMI. ${ }^{1}$ When young patients are analyzed, a great proportion is free from significant atherosclerotic lesions which indicates that different mechanisms can lead to coronary obstruction. ${ }^{2}$ Nonatherosclerotic coronary disease, hypercoagulable states and chemical substance abuse could also trigger an ischemic event. ${ }^{2}$ Also, the diagnosis of coronary dissection is more common in 
young women than men, ${ }^{4}$ and the artery most affected by dissection is the anterior descending artery. ${ }^{5}$

Findings in the literature correlated with this case reveal that the most common type of AMI is a single vessel disease, affecting mainly the left coronary artery in its anterior descending branch. ${ }^{6}$ The AFIJI cohort also demonstrated that a new ischemic event was caused more frequently by a new coronary lesion. ${ }^{3}$

Invasive and non-invasive imaging tests can be used to identify atherosclerosis. ${ }^{7}$ According to the 2019 ACC/AHA guidelines, coronary artery calcium (CAC) assessment is useful to estimate the risk. ${ }^{7}$ Also, coronary computed tomography has a potential role to guide prevention recommendations in younger adults. ${ }^{8}$ Advances in coronary computed tomography angiography has enabled the description of remodeling and necrotic aspects of plaques along with the association with the risk of coronary events. ${ }^{9}$

Regarding the prognosis of very young patients with STEMI, in-hospital mortality and survival rate are better compared with older patients. ${ }^{1,2}$ Recent findings have shown no difference in all-cause in-hospital mortality between young men and women. ${ }^{10}$ Although early mortality rate is low, additional ischemic events may occur in the future. ${ }^{3}$ In the 20-year cohort study, ${ }^{3}$ AMI was the most frequent new major acute cardiovascular event (MACE) (2.6 per 100 patient-years). All-cause death and stroke were less frequent in this group (1.60 per 100 patient-years, 0.70 per 100 patient-years, respectively). ${ }^{3}$

Although some risk factors for STEMI cannot be avoided, such as the family history, other risk factors can be modified. ${ }^{2}$ Discontinuance of cigarette smoking, for example, is the lifestyle change most likely to reduce the risk of a new ischemic event. ${ }^{3}$ In addition, hypertension and diabetes are well-known risk factors for CAD and should be treated in the young patient with previous STEMI to prevent recurrence. ${ }^{3}$ The AFIJI study also stated that LDL levels in these young patients was particularly higher when compared to the population that is most affected with STEMI. ${ }^{3}$ These results differ from the ones shown in this case report.

\section{Conclusion}

AMI in young adults remains a challenge in the emergency department. In this clinical case, although major risk factors for AMI were not evident, it is possible that other underlying mechanisms may have participated in this episode. More research is needed to evaluate chronic inflammatory profile in patients with premature coronary disease.

\section{Potential Conflict of Interest}

No potential conflict of interest relevant to this article was reported.

\section{Sources of Funding}

There were no external funding sources for this study.

\section{Study Association}

This study is not associated with any thesis or dissertation work.

\section{Ethics Approval and Consent to Participate}

This article does not contain any studies with human participants or animals performed by any of the authors.

\section{Author Contributions}

Conception and design of the research: Nascimento, EA. Acquisition of data: Mello, ALFP; Cunha, LS e Villela, MF. Analysis and interpretation of the data: Writing of the manuscript: Nascimento, EA; Mello, ALFP e Cunha, LS. Critical revision of the manuscript for intellectual content: Nascimento, EA. 


\section{References}

1. Puricel S, Lehner C, Oberhänsli M, Rutz T, Togni M, Stadelmann M, et al Acute coronary syndrome in patients younger than 30 years - aetiologies, baseline characteristics and long-term clinical outcome". Swiss Med Wkly. 2013; 143 .

2. Lisowska A, Makarewicz-Wujec M, Filipiak KJ. Risk factors, prognosis, and secondary prevention of myocardial infarction in young adults in Poland. Kardiol Pol. 2016;74:1148-53.

3. Collet, J-P, Zeitouni M, Procopi N, Hulot JS, Silvain J, Kernais M, et al. Long-Term Evolution of Premature Coronary Artery Disease. J Am Coll Cardiol 2019; 74(15):1868-78.

4. Vautrin E, Jean ABP, Fourny M, Marlière S, Vanzetto G, Bouvaist H, et al. Sex differences in coronary artery lesions and in-hospital outcomes for patients with ST-segment elevation myocardial infarction under the age of 45. Catheter Cardiovasc Interv. 2019 Dec;5 Doi: 10.1002/ccd:28627. Ahead print

5. Andrade HA, Feijó LA, Lavall GC, Tedeschi AL. Infarto agudo do miocárdio como apresentação de dissecção espontânea coronariana. Rev Bras Cardiol. 2010; 23(4):251-4.
6. Colkesen AY, Acil T, Demircan S, Sezgin AT, Muderrisoglu H. Coronary lesion type, location, and characteristics of acute ST elevation myocardial infarction in young adults under 35 years of age. Coron Artery Dis. 2008 Aug;19(5):345-7.

7. Arnett DK, Blumenthal RS, Albert MA, Buroker A, Goldberger Z, Hahn E, et al. 2019 ACC/AHA guideline on the primary prevention of cardiovascular disease: executive summary: a report of the American College of Cardiology/ American Heart Association Task Force on Clinical Practice Guidelines. J Am Coll Cardiol. 2019;74(10):1376-414.

8. Lin FY, Villines TC, Narula J, Shaw LJ. “What is the clinical role of noninvasive atherosclerosis imaging?". J Cardiovasc Comput Tomogr 2019 Jun;13(5):261-6.

9. Motoyama S, Sarai M, Harigaya H, Anno H, Inoue K, Hara T, et al. Computed tomographic angiography characteristics of atherosclerotic plaques subsequently resulting in acute coronary syndrome. J Am Coll Cardiol. 2009;54(1);49-57.

10. Bandyopadhyay D, Chakraborty S, Amgai B, Patel N, Hajra A, Heise $\mathrm{L}$,et al. Acute myocardial infarction in the young - National Trend Analysis with gender-based difference in outcomes. Int J Cardiol. 2020 Feb 15;301:21-8. 